\title{
Resveratrol increases the sensitivity of multiple myeloma cells against bortezomib via Hedgehog signaling pathway
}

\author{
Riyong Zhou ${ }^{1}$, Ruye $\mathrm{Ma}^{2}$, Zhenlin $\mathrm{Jin}^{3}$, Liyuan Tang ${ }^{3}$, Ying Zhou ${ }^{4}$, Yu Zhang ${ }^{3 *}$ \\ ${ }^{1}$ Department of Anesthesiology, The First Affiliated Hospital of Wenzhou Medical University, Wenzhou, Zhejiang 325015, \\ ${ }^{2}$ Department of Hematology, Jinshan Hospital of Fudan University, Jinan, Shandong 201500, ${ }^{3}$ Department of Hematology, The \\ First Affiliated Hospital of Wenzhou Medical University, Wenzhou, Zhejiang, 325015, ${ }^{4}$ Department of Nephrology, The First \\ Affiliated Hospital of Wenzhou Medical University, Wenzhou, Zhejiang 325015, China
}

*For correspondence: Email: YuZhangsdf@163.com; Tel: +86-577-55579481

\begin{abstract}
Purpose: To investigate the effect of resveratrol (RSV) on bortezomib (BTZ)-resistant multiple myeloma (MM) cells, and to elucidate the underlying mechanism of action.

Methods: H929 cell lines were exposed to BTZ for 8 months to establish BTZ-resistant MM cell model. Cell proliferation was determined by 3-(4,5-dimethylthiazol-2-yl)-2,5-diphenyltetrazolium bromide (MTT) assay. Apoptosis was measured using annexin V/propidium iodide (PI) staining while cell cycle analysis was evaluated by flow cytometry. The expression of Hedgehog $(H h)$ signaling proteins (sonic hedgehog $(S H H)$, smoothened (SMO), and glioma-associated oncogene homolog (GLI)) was analyzed by western blot.

Results: H929R was confirmed as a MM cell line that is resistant to BTZ. RSV enhanced the sensitivity of H929R cells against BTZ via inhibition of cell viability and colony formation, induction of cell apoptosis and regulation of expression of apoptosis-related proteins. Furthermore, RSV inhibited the expression of Hh signaling proteins $(p<0.05$.

Conclusion: RSV enhances the sensitivity of MM cells to BTZ, partly via Hh signaling pathway. Thus, Hh pathway is a probable target for MM treatment, and RSV has potentials for use in the clinical management of $M M$.
\end{abstract}

Keywords: Resveratrol, Bortezomib, Hedgehog signaling pathway, Multiple myeloma

\begin{abstract}
This is an Open Access article that uses a fund-ing model which does not charge readers or their institutions for access and distributed under the terms of the Creative Commons Attribution License (http://creativecommons.org/licenses/by/4.0) and the Budapest Open Access Initiative (http://www.budapestopenaccessinitiative.org/read), which permit unrestricted use, distribution, and reproduction in any medium, provided the original work is properly credited.
\end{abstract}

Tropical Journal of Pharmaceutical Research is indexed by Science Citation Index (SciSearch), Scopus, International Pharmaceutical Abstract, Chemical Abstracts, Embase, Index Copernicus, EBSCO, African Index Medicus, JournalSeek, Journal Citation Reports/Science Edition, Directory of Open Access Journals (DOAJ), African Journal Online, Bioline International, Open-J-Gate and Pharmacy Abstracts

\section{INTRODUCTION}

Multiple myeloma (MM), is characterized by clonal proliferation of malignant plasma cells, ranking the second most common hematological malignan [1]. In the past decades, supportive care strategies and new therapeutic agents (such as thalidomide, bortezomib (BTZ), and lenalidomide) have made significant breakthroughs in the treatment for MM[2]. However, MM is still incurable due to the resistance to drugs, particularly, BTZ). The mechanisms underlying the resistance against BTZ are complex, such as chromosomal translocations, oncogene mutations and disordered signaling pathways. Among them, Alonso et al reported that crosstalk between Hedgehog $(\mathrm{Hh})$ and retinoid signaling alters the 
MM microenvironment and generates BTZ resistance[3]. Other molecules are also involved the resistance against BTZ, including deubiquitinylating enzyme, ubiquitin receptor, serine synthesis, and Notch [4].

The Hh signaling pathway regulates the development and differentiation of tissues and organs during embryonic life. In mammals, this pathway can be activated when one of three ligands [Desert hedgehog, Indian hedgehog, or Sonic hedgehog $(\mathrm{SHH})]$ bind to their receptors (Patched)[5]. Hedgehog receptor PTCH then relieve smoothened (SMO) and result in the translocation of the glioma-associated oncogene homolog (GLI) and the transcription of Hh target genes[6].Studies have reported that the dysregulation of the Hh pathway may lead to tumorigenesis, including hepatic carcinoma, lung cancer, pancreatic cancer, and MM [7]. Beside, activation of $\mathrm{Hh}$ signaling is associated with drug resistance of MM stem cells, and the drug resistance is inhibited by $\mathrm{Hh}$ blocking agents[8].

Resveratrol (RSV), abundant in grapes and grape products, was widely used as an antioxidant and anti-mutagenic agent [9] . It exerts many beneficial effects, including antiinflammatory, antioxidant, cardiac protection, and anti-tumor and chemo-protective effects [10]. Previous studies reported that RSV inhibits the proliferation and invasion of MM cells, as well as cell cycle arrest and apoptosis [11]. Besides, in MM cells, resveratrol sensitizes apoptosis induced by carfilzomib via regulating oxidative stress [12]. Researchers reported the role of RSV in chemosensitization of cancers was associated with the modulation of drug efflux transporters, cell cycle, and autophagy through NF-KB and STAT pathways [13]. However, it is still unclear whether RSV has an effect on the resistance of MM against BTZ. Thus, the aim of present study was to characterize the effects of RSV on the chemoresistance against BTZ, and to identify the detail mechanism for this process.

\section{EXPERIMENTAL}

\section{Cell culture}

The H929 human MM cell line $\left(\right.$ ATCC $^{\circledR}$ CRL9068) was purchased from the American Type Culture Collection (Manassas, VA, USA). Bortezomib-resistant MM line was obtained by H929 exposure to BTZ for 8 months, with final concentration from 10 to $200 \mathrm{nM}$. The RPMI1640 medium (10\% fetal bovine serum) was changed twice each week by $50 \%$. In this manner, the concentration was gradually increased to $200 \mathrm{nM}$, and H929R cells resistant to bortezomib were obtained after 8 months of culture.

\section{Clonogenic assay}

Clonogenic assays were performed as reported [14]. Cells were cultured with 10\% FBS for $24 \mathrm{~h}$ and then with basal medium (containing $0.5 \%$ FBS) for 3 weeks, and then stained with $0.5 \%$ Crystal Violet solution, followed by phosphatebuffered saline to remove excess dye. Each treatment was performed in triplicate.

\section{MTT assay}

The principle of the MTT assay is that dehydrogenase enzyme in metabolically active cells converts the MTT dye (Sigma, St. Louis, $\mathrm{MO}$ ) to formazan crystals which are soluble in dimethyl sulfoxide (DMSO), which is quantified using a spectrophotometer. Cells were seeded in a 96-well plate to adhere overnight and treated with or without RSV for $48 \mathrm{~h}$. Then, $20 \mu \mathrm{L}$ of MTT solution was added and incubated for another 4 h. DMSO $(150 \mu \mathrm{L})$ was used to dissolve the formazan, and then measured at $570 \mathrm{~nm}$.

\section{Cell cycle analysis}

Cells were fixed in cold $70 \%$ alcohol overnight, permeabilized for 5 min with Triton X-100, and stained in a propidium iodide $(\mathrm{PI})$ solution with a final concentration of $50 \mu \mathrm{g} / \mathrm{mL}$ (Sigma-Aldrich, St. Louis, MO, USA). The analysis was performed using a FACScan flow cytometer (BD Biosciences, Bedford, MA, USA).

\section{Apoptosis analysis}

Cells were seeded at a density of $5 \times 10^{5}$ cells, and $24 \mathrm{~h}$ later, the medium was replaced and added with RSV or BTZ combined with RSV (the final concentration for RSV was 10 and $20 \mu \mathrm{M}$ for BTZ). The cells were collected after $72 \mathrm{~h}$, resuspended, then stained with annexin $\mathrm{V}$ and $\mathrm{PI}$ solution (BD Biosciences), and finally analyzed using flow cytometry.

\section{Western blot analysis}

Cells were seeded, treated with different methods, and collected. After being lysed in RIPA lysis buffer, protein was quantified using the BCA method and resolved using SDS-PAGE and transferred onto a PVDF membrane. After blocking, the membranes were incubated with primary antibodies[cleaved caspase 3 (Cell Signaling Technology, Inc. (CST), Danvers, MA, \#9661), cleaved caspase 9 (CST \#9505), BAX (CST \#5023), BCL-2 (CST \#15071), GLI-1 (CST 
\#2643), SHH (CST \#2207), and SMO (CST \#4930)using a dilution of 1:1,000; Cell Signaling Technology, Danvers, MA, USA] followed by incubation with secondary antibody $(1: 10,000)$, and detection of proteins using an ECL Western Blotting Analysis System (AmershamGEHealthcare, Little Chalfont, UK). The blots were standardized to glyceraldehyde 3-phosphate dehydrogenase.

\section{Statistical analysis}

One-way analysis of variance was used for statistical analysis. All data are expressed as mean \pm standard deviation (SD). Half maximal inhibitory concentration $\left(\mathrm{IC}_{50}\right)$ values were calculated using linear regression analysis.

\section{RESULTS}

\section{Confirmation of MM cell line (H929R) that resists to $\mathrm{BTZ}$}

H929 and H929R cells were cultured in media containing different concentrations of BTZ. The half-maximal inhibitory concentration $\left(\mathrm{IC}_{50}\right)$ was obtained using an MTT assay. Figure $1 \mathrm{~A}$ shows that BTZ effectively inhibited the proliferation of H929 cells $\left(\mathrm{IC}_{50}=13.45 \mathrm{nM}\right)$, but H929R cells were more resistant to $\mathrm{BTZ}\left(\mathrm{IC}_{50}=143.7 \mathrm{nM}\right)$. The colony formation results (Figure $1 \mathrm{~B}$ ) showed that the number of colonies formed by $\mathrm{H} 929$ cells was lower than that of H929R cells $(p<0.001)$, which was consistent with the MTT assay results. Thus, H929R cells were used in the following study.

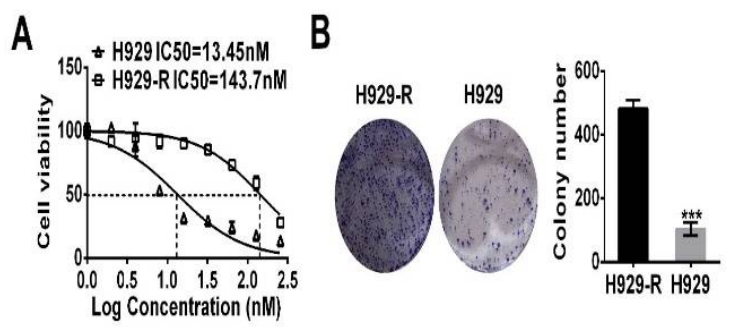

Figure 1: Viability and colony formation of bortezomib (BTZ)-treated H929R and H929 cells. (A) The viability of H929R and H929 cells treated with bortezomib (BTZ) measured using the MTT assay. (B) The colony formation of bortezomib (BTZ)-treated H929R and H929 cells analyzed using a clonogenic assay; ${ }^{* * *} p<0.001$ vs. H929-R cells

\section{RSV enhanced the sensitivity of H929R cells to BTZ}

To investigate the effects of RSV on the sensitivity of H929R cells against BTZ, H929R cells were treated with different concentration (5, 10,50 and $100 \mu \mathrm{M}$ ) of RSV or various exposure times $(12,24,36,48$ and $72 \mathrm{~h})$. The viability of H929R cells decreased as RSV concentration increased (Figure $2 \mathrm{~A}$ ). Viability was also decreased with longer incubation times (Figure 2 $B)$. The $I C_{50}$ of BTZ on H929R cells was decreased from 143.7 to $104.2 \mathrm{nM}$ (Figure $2 \mathrm{C}$ ). These results indicated that RSV enhanced the sensitivity of H929R cells against BTZ.

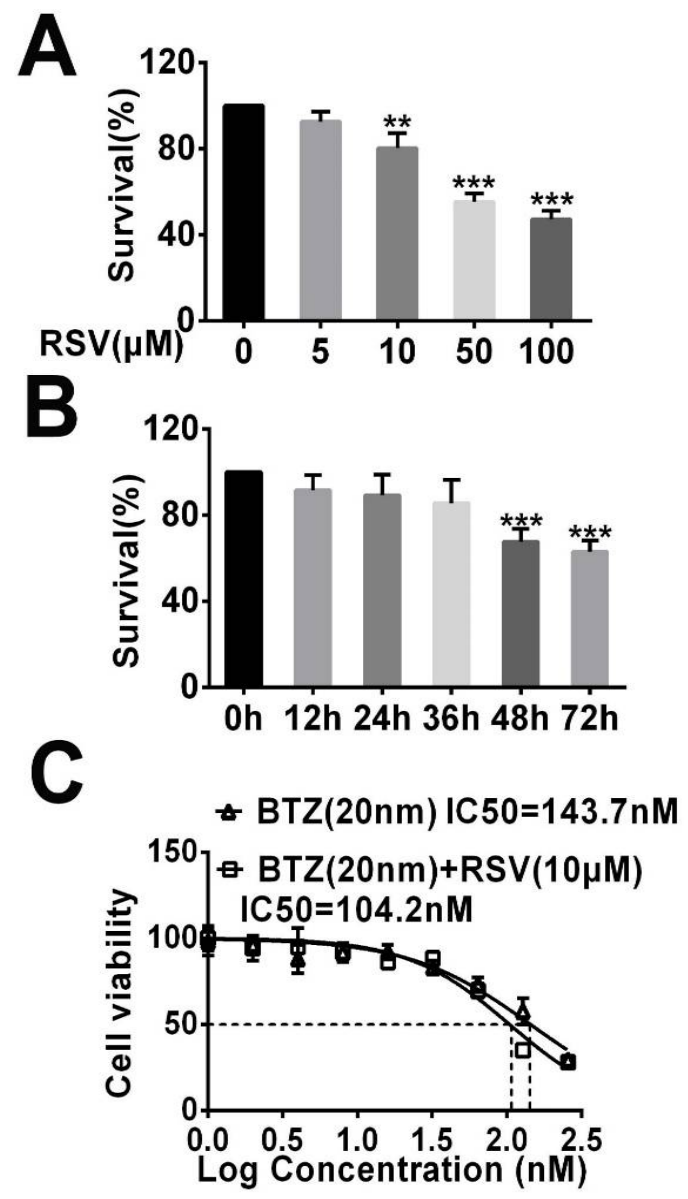

Figure 2: Effect of RSV on the viability of H929R cells. (A) The viability of H929R cells treated with different concentrations of RSV, followed by the MTT assay. (B) The viability of H929R cells with RSV treatment at various exposure periods, followed by the MTT assay. (C) The $\mathrm{IC}_{50}$ of BTZ on H929R cells. $p<0.05$ vs. bortezomib (BTZ) alone; $p<0.01$ and ${ }^{* * *} p<0.001$ vs. BTZ alone

RSV enhanced sensitivity of H929R cells to BTZ via inhibition of cell viability, colony formation and induction of cell apoptosis

For further analysis, proliferation, viability, colony formation, and cell cycle analysis of H929R cells were performed. Figure $3 \mathrm{~A}$ showed that BTZ did not inhibit the viability of H929R cells as compared with normal control group, confirming the resistant of H929R cells to BTZ. In contrast, RSV combined with BTZ treatment inhibited the proliferation of $\mathrm{H} 929 \mathrm{R}$ when compared with BTZ 
alone $(p<0.01)$, which indicated that RSV enhanced the sensitivity of H929R cells against BTZ through inhibiting cell viability. The colony formation results were consistent with the results of cell viability (Figure $3 \mathrm{~B}$ ). To explore the mechanism of RSV-inhibited cell proliferation, the cell cycle was analyzed using flow cytometry. The amount of G1, S, and G2phases are shown in Figure $3 \mathrm{C}$. The fraction in the $\mathrm{S}$ phase was lower than that of RSV+BTZ group compared to the BTZ group.

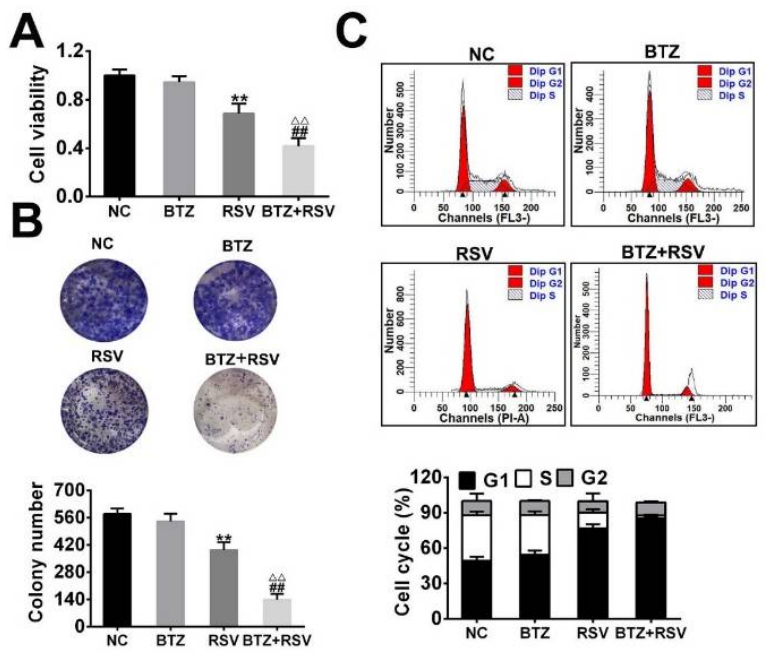

Figure 3: RSV enhanced the sensitivity of H929R cells against BTZ through inhibiting cell viabiliy and colony formation and inducing cell apoptosis. (A) The viability of H929R cells measuredusing the MTT assay. (B) The colony formation of H929R cells analyzed by the clonogenic assay. (C) The cell cycle was analyzed using flow cytometry; ${ }^{* *} p<0.01$ vs. NC; ${ }^{\# \#} p<0.01$ vs. bortezomib (BTZ); $\Delta \Delta p<0.01$ vs. RSV

\section{RSV enhanced BTZ-induced apoptosis of H929R cells}

As Resveratrol (RSV) enhanced the sensitivity of H929R against BTZ, the effects of RSV on the apoptosis of H929Rwere determined using annexin V/PI staining. Figure 4A showed that the proportion of apoptotic cells in the BTZ group was increased as compared to the normal control $(p<0.01)$. RSV combined with BTZ significantly increased the number of apoptotic cells $(p<0.01$ vs. BTZ). To further verify the effects of RSV on BTZ-induced apoptosis of H929R, apoptosisrelated proteins were measured using western blot analysis. Figure 4B showed that BTZ elevated the expression levels of activated caspases 3 and 9 (cleaved caspase 3 and 9 ) and a pro-apoptotic protein (BAX). Addition of RSV aggravated these results as compared with BTZ alone.

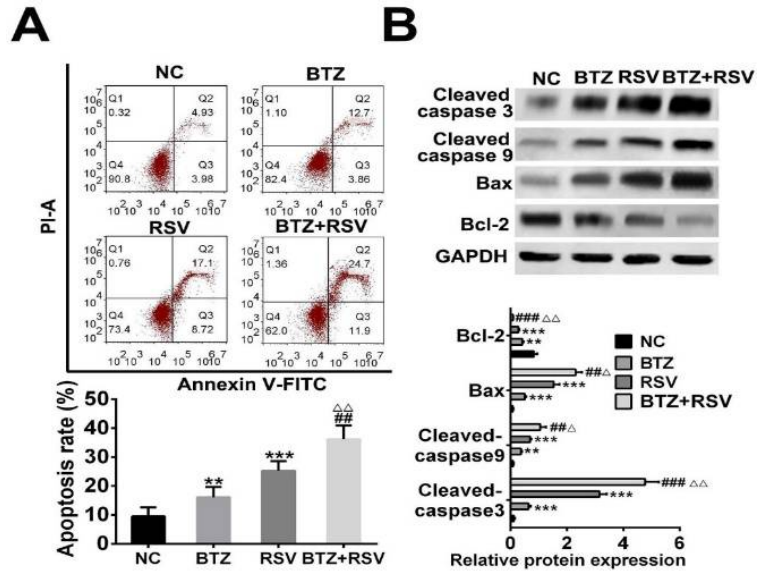

Figure 4: Resveratrol (RSV) enhanced the apoptosis of H929R cells.(A) Cell apoptosis by annexin V/PI staining. Q1: annexin-/PI+ necrotic cells, Q2: annexin+/PI+ late apoptotic cells, Q3: annexin-/PIviable cells, Q4: annexin +/PI- early apoptotic cells. (B) The level of apoptosis-related proteins was determined using western blotting; $p<0.01$ and ${ }^{* * *} p<$ 0.001 vs. normal control; ${ }^{\# \#} p<0.01$ and ${ }^{\# \# \#} p<0.001$ vs. bortezomib (BTZ); $\Delta \Delta p<0.01$ and $\Delta \Delta \Delta p<0.001$ vs. RSV

\section{RSV inhibited the activation of the Hh signaling pathway of H929R cells}

To reveal the underlying mechanism, $\mathrm{Hh}$ signaling proteins $(\mathrm{SHH}, \mathrm{SMO}$, and $\mathrm{GLI}-1)$ were analyzed using western blotting. Figure 5 showed that BTZ alone did not affect the expression of these three proteins, while RSV inhibited the expression of $\mathrm{SHH}(p<0.001)$, SMO $(p<0.001)$, and GLI-1 $(p<0.01)$. RSV, when combined with BTZ, enhanced the effects of RSV on H929R cells, which indicated that RSV enhanced the sensitivity of H929R cells to BTZ partly through the Hh signaling pathway.

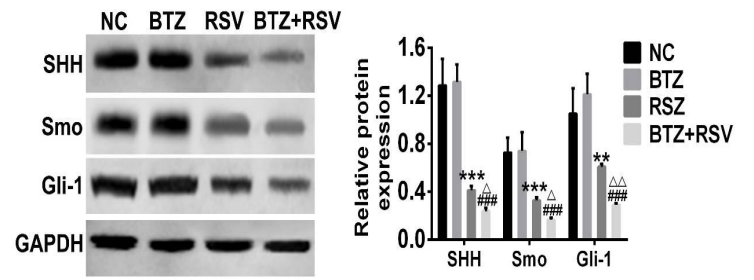

Figure 5: Effect of RSV on expression of Hedgehog signaling proteins $(\mathrm{SHH}, \mathrm{SMO}$, and $\mathrm{GLI}-1)$ analyzed by western blot; $p<0.01$ and ${ }^{* * *} p<0.001$ vs. normal control; ${ }^{\# \#} p<0.001$ vs. bortezomib (BTZ); $\Delta p<0.05$ and $\Delta \Delta p<0.01$ vs. RSV

\section{DISCUSSION}

Multiple myeloma (MM) is the second most common type of hematological cancer, with median survival of 3-4 years from the initial 
diagnosis. Although the development of newly marketed proteasome inhibitors and immune modulators has improved the effectiveness of treatment, MM remains incurable for patients. Besides, chemotherapy resistance is still one of the important causes for failure of treatment. Studies have indicated that the use of Chinese Material Medica in clinical practice has achieved good effects in the treatment of MM. Resveratrol (RSV), a major active compound of stilbene phytoalexins, has been proven to be beneficial for health. The biological effects of RSV are diverse, including antioxidant activity, modulation of lipid and lipoprotein metabolism, antiplatelet aggregation, anticancer and estrogenic activity [15].

Studies have reported that RSV could be a novel agent for the treatment of MM. Researchers [12] found that RSV sensitized proteasome inhibitorinduced apoptosis via regulating oxidative stress in MM cells. Similarly, Jazirehi et al [16] reported that RSV sensitized paclitaxel-induced apoptosis by modifying the expression of apoptotic proteins and regulating the cell cycle. Other researchers investigated the mechanism of RSV on MM cell proliferation, apoptosis, and chemoresistance. Their results indicated that RSV inhibited MM cell proliferation, promoted apoptosis, and decreased chemoresistance via downregulation of STAT3 and nuclear factor-B-regulated anti-apoptotic gene expression [17].

Consistently, the present study confirmed the previous results that RSV sensitizes MM cells to BTZ (a proteasome inhibitor).Firstly, a BTZresistant MM cell line (H929R) was established to further verify the effects of RSV on the resistance of MM cells against BTZ. The results showed that RSV enhanced the sensitivity of H929R cells against BTZ through inhibiting cell viabiliy and colony formation, inducing cell apoptosis and regulating the expression of apoptosis-related proteins.

The mechanism of MM generation and development is complex, and it is very important to elucidate the pathogenesis of MM or the effect of drugs on the clinical treatment. Previous studies found that Wnt, STAT3, NF-KB , MAPK, $A K T$, and the $\mathrm{Hh}$ signaling pathway were involved in the proliferation, apoptosis, cell invasion, and chemoresistance of MM [18]. Particuarly, Hh signaling pathway, a highly conserved system which regulates the development and differentiation of tissues, was recently confirmed to be associated with the progression of MM [19].

A phase I clinical study of the Hh pathway antagonist (BMS-833923) in patients with relapsed or refractory MM showed that inhibition of the Hh pathway effectively improved the treatment effect of MM patients[20]. Research also demonstrated that the Hh signaling pathway plays a key role in MM. Hedgehog activation was heterogeneous across the spectrum of MM tumor stem cells, to maintain a tumor stem cell compartment [21].The inhibition of $\mathrm{Hh}$ signaling inhibited clonogenic growth and self-renewal in MM cells [22].These results showed that RSV improved the sensitivity of MM cells to BTZ via inhibiting the activation of the $\mathrm{Hh}$ signaling pathway, although few studies had reported this mechanism of RSV on BTZ-resistant in cells.

\section{CONCLUSION}

RSV enhanced the sensitivity of H929R cells to BTZ via inhibition of cell viabiliy and colony formation, induction of cell apoptosis and regulation of the expression of apoptosis-related proteins. The underlying mechanism of action is associated with $\mathrm{Hh}$ signaling pathway. Hh pathway is, therefore, a target for MM treatment, and thus, RSV may be developed as a candidate for the therapeutic management of MM.

\section{DECLARATIONS}

\section{Acknowledgement}

This work was supported by the Zhejiang Provincial Natural Science Foundation of China. (Grant nos. LQ19H080003 and LQ19H050002).

\section{Conflict of interest}

The authors declare that no conflict of interest is associated with this work.

\section{Contribution of authors}

We declare that this work was done by the researchers listed in this article. All liabilities related with the content of this article will be borne by the authors. $R Z$ and $Y Z$ designed all the experiments and revised the paper. RM and $Z J$ carried out the experiments. LT and $Y Z$ wrote the manuscript.

\section{Open Access}

This is an Open Access article that uses a funding model which does not charge readers or their institutions for access and distributed under the terms of the Creative Commons Attribution License (http://creativecommons.org/licenses/by/ 4.0) and the Budapest Open Access Initiative 
(http://www.budapestopenaccessinitiative.org/rea d), which permit unrestricted use, distribution, and reproduction in any medium, provided the original work is properly credited.

\section{REFERENCES}

1. Rajkumar SV. Multiple myeloma: 2016 update on diagnosis, risk-stratification, and management. American Journal of Hematology 2016; 91(7): 719-734.

2. Palumbo A, Chanankhan A, Weisel K, Nooka AK, Masszi $T$, Beksac M, Spicka I, Hungria V, Munder M, Mateos MV. Daratumumab, Bortezomib, and Dexamethasone for Multiple Myeloma. New England Journal of Medicine 2016; 375(8): 754-766.

3. Alonso S, Hernandez D, Chang Y-t, Gocke CB, McCray $M$, Varadhan R, Matsui WH, Jones RJ, Ghiaur GJTJoci. Hedgehog and retinoid signaling alters multiple myeloma microenvironment and generates bortezomib resistance. The Journal of clinical investigation 2017; 126(12): 4460-4468.

4. Song $Y$, Ray A, Chauhan D, Anderso K. Blockade of Ubiquitin Receptor PSMD4/Rpn10 Triggers Cytotoxicity and Overcomes Bortezomib-Resistance in Multiple Myeloma. The Journal of clinical investigation 2018; 132(Suppl 1): 3211.

5. Simona $B$, Jana J, Teresa $C$, Roccaro $A M$, Nicola $A$, Abdel Kareem A, Umberto F, Mitsiades CS, Marco R, Katia T. Canonical and noncanonical Hedgehog pathway in the pathogenesis of multiple myeloma. Blood 2012; 120(25): 5002.

6. Wang C, Zhu M, Lu X, Wang H, Zhao W, Zhang X, Dong $X$. Synthesis and evaluation of novel dimethylpyridazine derivatives as hedgehog signaling pathway inhibitors. Bioorganic \& Medicinal Chemistry 2018.

7. Chung SI, Moon H, Ju HL, Cho KJ, Kim DY, Han KH, Eun JW, Nam SW, Ribback S, Dombrowski F. Hepatic expression of Sonic Hedgehog induces liver fibrosis and promotes hepatocarcinogenesis in a transgenic mouse model. Journal of Hepatology 2016; 64(3): 618-627.

8. Gao M, Kong Y, Yang G, Gao L, Shi J. Multiple myeloma cancer stem cells. Oncotarget 2016; 7(23): 3546635477.

9. Barreto DC, Gomez RS, Bale AE, Boson WL, De Marco L. PTCH Gene Mutations in Odontogenic Keratocysts. 2000; 79(6): 1418-1422.

10. Zhang $X$, Yang $Z$, Li L, Qiao Y, Jiao H, Miao C. Prevention of injury by resveratrol in a rat model of adenine-induced chronic kidney disease Tropical Journal of Pharmaceutical Research 2017; 16(8): 20272032.

11. Chunyan, Hu, Yu, Liu, Xinyue, Wu, Tao, Wang, Yadan. Resveratrol downregulates the constitutional activation of nuclear factor-KB in multiple myeloma cells, leading to suppression of proliferation and invasion, arrest of cell cycle, and induction of apoptosis. Cancer Genetics \& Cytogenetics 2006; 165(1): 9-19.

12. Li $Q$, Yue $Y$, Chen $L, X u C$, Wang $Y$, Du L, Xue $X$, Liu $Q$, Fan F. Resveratrol Sensitizes Carfilzomib-Induced Apoptosis via Promoting Oxidative Stress in Multiple Myeloma Cells. Front Pharmacol 2018; 9(334.

13. Mohammed S, Harikumar KB: Chapter 3 - Role of Resveratrol in Chemosensitization of Cancer. In: Role of Nutraceuticals in Cancer Chemosensitization. Edited by Bharti AC, Aggarwal BB, vol. 2: Academic Press; 2018: 61-76.

14. Bolin L, Dalia OE, Zeying F, Edgerton SM, Xiaohe Y, Thor AD. Downregulation of erbB3 abrogates erbB2mediated tamoxifen resistance in breast cancer cells. International Journal of Cancer 2010; 120(9): 18741882.

15. Frémont $L$. Biological effects of resveratrol. Life Sciences 2000; 66(8): 663-673.

16. Jazirehi AR, Bonavida B. Resveratrol modifies the expression of apoptotic regulatory proteins and sensitizes non-Hodgkin's lymphoma and multiple myeloma cell lines to paclitaxel-induced apoptosis. Mol Cancer Ther 2004; 3(1): 71-84.

17. Bhardwaj A, Sethi G, Vadhan-Raj S, Bueso-Ramos C, Takada Y, Dixit U, Nair A, Shishodia S, Aggarwal B. Resveratrol inhibits proliferation, induces apoptosis, and overcomes chemoresistance through down-regulation of STAT3 and nuclear factor-kappaB-regulated antiapoptotic and cell survival gene products in human multiple myeloma cells. Blood 2007; 109(2293-2302.

18. Kim JH, Park B. Triptolide blocks the STAT3 signaling pathway through induction of protein tyrosine phosphatase SHP-1 in multiple myeloma cells. International Journal of Molecular Medicine 2017; 40(5): 1566-1572.

19. Peacock $C D$, Wang $Q$, Gesell GS, Corcoran-Schwartz IM, Jones E, Kim J, Devereux WL, Rhodes JT, Huff CA, Beachy $P A$ et al. Hedgehog signaling maintains a tumor stem cell compartment in multiple myeloma. Proceedings of the National Academy of Sciences 2007; 104(10): 4048-4053.

20. Huff, Carol A. A Phase I Study of An Oral Hedgehog Pathway Antagonist, BMS-833923, in Patients with Relapsed or Refractory Multiple Myeloma. Blood 2011; 118(21): 3993.

21. Peacock $C D$, Wang $Q$, Gesell GS, Corcoran-Schwartz IM, Jones E, Kim J, Devereux WL, Rhodes JT, Huff CA, Beachy PA et al. Hedgehog signaling maintains a tumor stem cell compartment in multiple myeloma. Proc Natl Acad Sci U S A 2007; 104(10): 4048-4053.

22. Agarwal JR, Qiuju W, Toshihiko T, Zeshaan R, Akil M, Nilanjan G, Ivan B, Carol Ann H, Farhad P, William M. Activation of liver $X$ receptors inhibits hedgehog signaling, clonogenic growth, and self-renewal in multiple myeloma. Mol Cancer Ther 2014; 13(7): 18731881. 